\title{
Four-dimensional integration by parts with differential renormalization as a method of evaluation of Feynman diagrams
}

\author{
V.A. Smirnov円 \\ Nuclear Physics Institute of Moscow State University \\ Moscow 119899, Russia
}

\begin{abstract}
It is shown how strictly four-dimensional integration by parts combined with differential renormalization and its infrared analogue can be applied for calculation of Feynman diagrams.
\end{abstract}

submitted to Teor. Mat. Fiz.

${ }^{1}$ E-mail: smirnov@theory.npi.msu.su 
1. Integration by parts (IBP) [1] within dimensional regularization [2] turned out to be one of the most powerful methods of calculation of Feynman diagrams. In spite of the fact that dimensional regularization and renormalization [3] are commonly used in practical calculations they happen to be inconvenient in situations where chiral and super symmetries are involved. The purpose of this brief note is to present a strictly four-dimensional version of this method of calculation. It will be shown how (usual) four-dimensional IBP supplied with technique of differential renormalization [4-9] and its infrared (IR) analogue ( $\tilde{R}$-operation) can be used for calculation of diagrams.

In the next section we shall describe prescriptions of differential renormalization and $\tilde{R}$-operation for simplest diagrams. Using an example of the master two-loop diagram, we shall show in Section 3 how four-dimensional IBP is applied for calculations. In conclusion possible applications of the proposed method are applied.

2. Differential renormalization was first defined in coordinate space [4-8]. It is possible to translate its recipes into the momentum space language. Another possibility is to use homogeneity properties of Feynman integrals and formulate the corresponding recipe just in momentum space - see [9] where this prescription was formulated for logarithmically divergent diagrams with the simplest topology of subdivergences. (Probably, it is more natural to call this renormalization homogeneous rather than differential.) In particular, for the simplest one-loop propagator-type Feynman integrall one has the following renormalization prescription [9]:

$$
R F(q) \equiv R \int \mathrm{d}^{4} k \frac{1}{k^{2}(q-k)^{2}}=\int \mathrm{d}^{4} k \ln k^{2} / \mu^{2} \frac{1}{2} q \frac{\partial}{\partial q} \frac{1}{k^{2}(q-k)^{2}} .
$$

Here $\mu$ is a massive parameter that takes into account finite arbitrariness of renormalization. The derivative in $q$ is applied before integration so that the corresponding integral happens to be convergent.

To calculate (11) it is worthwhile to introduce analytic regularization [10]:

$$
R F(q)=\left.\int \mathrm{d}^{4} k\left(-\frac{\mathrm{d}}{\mathrm{d} \lambda}\right) \frac{1}{2} q \frac{\partial}{\partial q} \frac{\mu^{2 \lambda}}{\left(k^{2}\right)^{1+\lambda}(q-k)^{2}}\right|_{\lambda=0} .
$$

When $\lambda \neq 0$, we may use the following order: to calculate the integral, differentiate in $q$, differentiate in $\lambda$ and finally put $\lambda=0$. When calculating the integral one uses the four-dimensional one-loop formula

$$
\int \mathrm{d}^{4} k \frac{1}{\left(k^{2}\right)^{\lambda_{1}}\left((q-k)^{2}\right)^{\lambda_{2}}}=\pi^{2} G\left(\lambda_{1}, \lambda_{2}\right) \frac{1}{\left(q^{2}\right)^{\lambda_{1}+\lambda_{2}-2}},
$$

where $G$ is the four-dimensional $G$-function

$$
G\left(\lambda_{1}, \lambda_{2}\right)=\frac{\Gamma\left(\lambda_{1}+\lambda_{2}-2\right)}{\Gamma\left(\lambda_{1}\right) \Gamma\left(\lambda_{2}\right)} \frac{\Gamma\left(2-\lambda_{1}\right) \Gamma\left(2-\lambda_{2}\right)}{\Gamma\left(4-\lambda_{1}-\lambda_{2}\right)} .
$$

\footnotetext{
${ }^{2}$ For simplicity, we consider Feynman integrals in Euclidean space.
} 
In particular,

$$
G(1,1+\lambda)=\frac{1}{\lambda(1-\lambda)}
$$

Finally we have

$$
R \int \mathrm{d}^{4} k \frac{1}{k^{2}(q-k)^{2}}=\pi^{2}\left(1-\ln q^{2} / \mu^{2}\right) .
$$

We shall need as well an IR analogue of differential renormalization. For the case of dimensional renormalization, the corresponding IR analogue was developed in [11]. This operation $\tilde{R}$ removes IR divergences (off the mass shell) in a quite similar way as usual dimensional renormalization removes ultraviolet (UV) divergences. When we combine it with the $R$-operation itself we obtain the so-called $R^{*}$-operation [11] $\left(R^{*}=\tilde{R} R\right)$ which is successfully applied in renormalization group calculations and in asymptotic expansions of Feynman integrals in various limits of momenta and masses (see. e.g., [12] for a review).

Differential $\tilde{R}$-operation can be defined in momentum space in quite the same way as differential renormalization in coordinate space. ${ }^{3}$ For the simplest IR-divergent subgraph consisting of two subsequent massless lines that generate a nonintegrable factor $1 / k^{4}$, we have the following prescription:

$$
\tilde{R} \frac{1}{k^{4}}=\frac{1}{2} \frac{\partial}{\partial k} k\left(\frac{\ln k^{2} / \tilde{\mu}^{2}}{k^{4}}\right),
$$

where $\tilde{\mu}$ - is an IR-renormalization parameter. Therefore, for the one-loop IR-divergent Feynman integral

$$
\int \mathrm{d}^{4} k \frac{1}{k^{4}(q-k)^{2}}
$$

we obtain the following ' $\tilde{R}$-normalized' expression:

$$
\tilde{R} \int \mathrm{d}^{4} k \frac{1}{k^{4}(q-k)^{2}}=\int \mathrm{d}^{4} k\left(\frac{1}{2} \frac{\partial}{\partial k} k \frac{\ln k^{2} / \tilde{\mu}^{2}}{k^{4}}\right) \frac{1}{(q-k)^{2}} .
$$

The derivative involved in (7) and (8) is understood in the distributional sense (that is formally equivalent to the four-dimensional IBP). i.e.

$$
\tilde{R} \int \mathrm{d}^{4} k \frac{1}{k^{4}(q-k)^{2}}=\int \mathrm{d}^{4} k \frac{k(k-q) \ln k^{2} / \tilde{\mu}^{2}}{k^{4}(q-k)^{4}} .
$$

To calculate (8) it is helpful to introduce analytic regularization and then differentiate the integrand at $\lambda \neq 0$ using $\frac{1}{2} \frac{\partial}{\partial k} k \frac{\tilde{\mu}^{2 \lambda}}{\left(k^{2}\right)^{2+\lambda}}=-\lambda \frac{\tilde{\mu}^{2 \lambda}}{\left(k^{2}\right)^{2+\lambda}}$. We have

$$
\tilde{R} \int \mathrm{d}^{4} k \frac{1}{k^{4}(q-k)^{2}}=\pi^{2} \frac{1}{q^{2}}\left(1+\ln q^{2} / \tilde{\mu}^{2}\right) .
$$

\footnotetext{
${ }^{3}$ To be more precise, renormalization prescriptions in coordinate space formulated in refs. 6 , 8 , are reformulated as 'IR-renormalization' prescriptions in momentum space, using UV-IR analogy (as it was done in the case of dimensional $\tilde{R}$-operation - see 11, 12]).
} 
We shall need also similar integrals with additional logarithms which are as well calculated with the help of analytic regularization and one-loop integration formula (3). One gets

$$
\begin{gathered}
\tilde{R} \int \mathrm{d}^{4} k \frac{\ln \left((q-k)^{2} / \mu^{2}\right)}{k^{4}(q-k)^{2}}=\int \mathrm{d}^{4} k\left(\frac{1}{2} \frac{\partial}{\partial k} k \frac{\ln \left(k^{2} / \tilde{\mu}^{2}\right)}{k^{4}}\right) \frac{\ln (q-k)^{2} / \mu^{2}}{(q-k)^{2}} \\
=\pi^{2} \frac{1}{q^{2}} \ln q^{2} / \mu^{2}\left(\ln q^{2} / \tilde{\mu}^{2}+1\right) ; \\
\tilde{R} \int \mathrm{d}^{4} k \frac{\ln k^{2} / \tilde{\mu}^{2}}{k^{4}(q-k)^{2}}=\int \mathrm{d}^{4} k\left(\frac{1}{4} \frac{\partial}{\partial k} k \frac{\ln ^{2} k^{2} / \tilde{\mu}^{2}}{k^{4}}\right) \frac{\ln (q-k)^{2} / \mu^{2}}{(q-k)^{2}} \\
=\pi^{2} \frac{1}{q^{2}}\left(1+\ln q^{2} / \tilde{\mu}^{2}+\frac{1}{2} \ln ^{2} q^{2} / \tilde{\mu}^{2}\right) ; \\
\tilde{R} \int \mathrm{d}^{4} k \frac{\ln ^{2}\left((q-k)^{2} / \mu^{2}\right)}{k^{4}(q-k)^{2}}=\int \mathrm{d}^{4} k\left(\frac{1}{2} \frac{\partial}{\partial k} k \frac{\ln k^{2} / \tilde{\mu}^{2}}{k^{4}}\right) \frac{\ln ^{2}(q-k)^{2} / \mu^{2}}{(q-k)^{2}} \\
q^{2}\left(\ln ^{2} q^{2} / \mu^{2}\left(\ln q^{2} / \tilde{\mu}^{2}+1\right)+4 \zeta(3)\right) ; \\
\tilde{R} \int \mathrm{d}^{4} k \frac{\ln \left((q-k)^{2} / \mu^{2}\right) \ln k^{2} / \tilde{\mu}^{2}}{k^{4}(q-k)^{2}}=\int \mathrm{d}^{4} k\left(\frac{1}{4} \frac{\partial}{\partial k} k \frac{\ln ^{2} k^{2} / \tilde{\mu}^{2}}{k^{4}}\right) \frac{\ln (q-k)^{2} / \mu^{2}}{(q-k)^{2}} \\
=\pi^{2} \frac{1}{q^{2}}\left(\ln q^{2} / \mu^{2}\left(1+\ln q^{2} / \tilde{\mu}^{2}+\frac{1}{2} \ln ^{2} q^{2} / \tilde{\mu}^{2}\right)+2 \zeta(3)\right) .
\end{gathered}
$$

Here $\tilde{\mu}$ is an IR renormalization parameter and $\zeta(z)$ Riemann zeta-function.

3. To calculate the two-loop master massless diagram (see Fig. 1) strictly in four dimensions

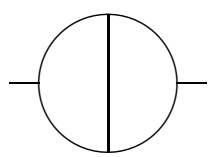

Figure 1: Two-loop master diagram.

$$
J(q)=\iint \frac{\mathrm{d}^{4} k \mathrm{~d}^{4} l}{k^{2}(k-q)^{2}(k-l)^{2} l^{2}(l-q)^{2}}
$$


let us apply four-dimensional IBP

$$
\iint \mathrm{d}^{4} k \mathrm{~d}^{4} l \frac{\partial}{\partial l_{\alpha}}\left\{\frac{(k-l)_{\alpha}}{k^{2}(k-q)^{2}(k-l)^{2} l^{2}(l-q)^{2}}\right\}=0 .
$$

Formally, this is the same identity that was used within the standard IBP method [1] based on dimensional regularization. Using relations $2(k-l) l=k^{2}-(k-l)^{2}-l^{2}$, $2(k-l)(l-q)=(k-q)^{2}-(k-l)^{2}-(l-q)^{2}$, one gets

$$
\iint \mathrm{d}^{4} k \mathrm{~d}^{4} l \frac{(k-l)^{2}-(k-q)^{2}}{k^{2}(k-q)^{2}(k-l)^{2} l^{2}(l-q)^{4}}=0 .
$$

Although this integral is UV and IR convergent one obtains divergences of both kind when considering separately two terms in the numerator. It is possible however to separate them by introducing (in advance) $R$ and $\tilde{R}$ operations. For example, for the $R$-operation one uses the following identities (with $j=1,2, \ldots$ ):

$$
\int \mathrm{d} k \ln ^{j} l^{2} / \tilde{\mu}^{2} \Pi(k, l, q)=\int \mathrm{d} k \ln k^{2} / \mu^{2} \ln ^{j} l^{2} / \tilde{\mu}^{2} \frac{1}{2}\left(q \frac{\partial}{\partial q}+l \frac{\partial}{\partial l}-\lambda-4\right) \Pi(k, l, q),
$$

where $\Pi$ is a homogenous function of $k, l, q$ of degree $\lambda$. Then one obtains

$$
R^{*} \iint \mathrm{d}^{4} k \mathrm{~d}^{4} l \frac{1}{k^{2}(k-q)^{2} l^{2}(l-q)^{4}}=R^{*} \iint \mathrm{d}^{4} k \mathrm{~d}^{4} l \frac{1}{k^{2}(k-l)^{2} l^{2}(l-q)^{4}}
$$

where $R^{*}=R \tilde{R}$.

Let us now apply four-dimensional IBP with additional logarithm:

$$
\iint \mathrm{d}^{4} k \mathrm{~d}^{4} l \frac{\partial}{\partial l_{\alpha}}\left\{\frac{(k-l)_{\alpha} \ln l^{2} / \tilde{\mu}^{2}}{k^{2}(k-q)^{2}(k-l)^{2} l^{2}(l-q)^{2}}\right\}=0 .
$$

After that one comes to

$$
\begin{array}{r}
J(q)=R \int \mathrm{d}^{4} k \frac{1}{k^{2}(q-k)^{2}} \tilde{R} \int \mathrm{d}^{4} l \frac{\ln \left((q-l)^{2} / \tilde{\mu}^{2}\right)}{l^{2}(q-l)^{4}} \\
+R \int \mathrm{d}^{4} k \frac{1}{k^{2}(q-k)^{2}} \tilde{R} \int \mathrm{d}^{4} l \frac{\ln l^{2} / \tilde{\mu}^{2}}{l^{2}(q-l)^{4}}-R^{*} \iint \mathrm{d}^{4} k \mathrm{~d}^{4} l \frac{\ln l^{2} / \tilde{\mu}^{2}}{k^{2}(k-l)^{2} l^{2}(q-l)^{4}} \\
-R^{*} \iint \mathrm{d}^{4} k \mathrm{~d}^{4} l \frac{\ln \left((l-q)^{2} / \tilde{\mu}^{2}\right)}{k^{2}(k-l)^{2} l^{2}(q-l)^{4}} .
\end{array}
$$

For evaluation of recursively one-loop Feynman integrals involved, one uses (6, 10 14) and arrives at the well-known result [13]

$$
J(q)=6 \zeta(3) \pi^{4} / q^{2} .
$$

4. As in the above example of the master two-loop diagram one can apply fourdimensional IBP with differential $R$ and $\tilde{R}$-operations at least in every situation where 
IBP within dimensional regularization is applied. To do this one uses almost the same identities as in the case of dimensional regularization. Roughly speaking, negative powers of $\epsilon=(4-d) / 2$ (where $d$ is the space-time dimension) are replaced by some powers of logarithms of the loop momenta. To be able to consider separately individual terms in these identities one removes artificial UV and IR divergences in advance (in the case of dimensional regularization they were automatically regularized). To perform further calculations one needs a table of one-loop integrals with logarithms (in the considered case, these are formulae (6, 10 14) ).

Note that within analytic regularization one fails to use IBP for calculation of the master diagram, as it is possible within dimensional regularization and, strictly in four dimensions, in the presented technique. This can be explained by impossibility of cancellations of the type $p^{2} / p^{2}=1$ in the integrands (because one obtains $p^{2}$ in denominators in a power dependent on the regularization parameter). Therefore, although the table of the one-loop integrals was derived with the help of analytic regularization, the logarithms involved do not spoil these cancellations (graphically, corresponding to contraction of lines to a point) which lead to recursively one-loop integrals in the right-hand side of (21).

This fact looks promising for construction of differential renormalization schemes compatible with the gauge invariance because Ward identities are finally connected with the possibility of the above cancellations in renormalized Feynman integrals. It turns out that it is possible to modify the prescriptions of differential renormalization of refs. [6, 8] in such a way that the gauge invariance will be preserved automatically (i.e. without adjusting finite counterterms) at least in the Abelian case. If This assertion will be justified in a separate paper.

Finally, let us stress that for applications in theories with chiral and super symmetries, the four-dimensional technique looks more preferable.

This work is supported by the Russian Foundation for Basic Research, project 9601-00654.

Acknowledgments. I am grateful to K.G. Chetyrkin and G.A. Kravtsova for valuable discussions.

\section{References}

[1] K.G. Chetyrkin and F.V. Tkachov, Nucl. Phys. B192 (1981) 159

[2] G. 't Hooft and M. Veltman, Nucl. Phys. B44 (1972) 189;

C.G. Bollini and J.J. Giambiagi, Nuovo Cim. 12B (1972) 20

[3] P. Breitenlohner and D. Maison, Commun. Math. Phys. 52 (1977) 11, 39, 55

[4] D.Z. Freedman, K. Johnson and J.I. Latorre, Nucl. Phys. B371 (1992) 353

\footnotetext{
${ }^{4}$ See also ref. [14] where relations relevant to Ward identities in QED were proved in the framework of differential renormalization for some special classes of diagrams.
} 
[5] J.I. Latorre, C. Manuel and X. Vilasís Cardona, Ann. Phys. 231 (1994) 149

[6] V.A. Smirnov and O.I. Zavialov, Teor. Mat. Fiz. 96 (1993) 288 [Theor. Math. Phys. 96 (1993) 974]

[7] V.A. Smirnov, Nucl. Phys. B427 (1994) 325

[8] V.A. Smirnov, Z. Phys. C 67 (1995) 531

[9] O.I. Zavialov, Teor. Mat. Fiz. 98 (1994) 536

[10] E.R. Speer, J. Math. Phys. 9 (1968) 1404

[11] K.G. Chetyrkin and V.A. Smirnov, Phys. Lett. 144B (1984) 419

[12] V.A. Smirnov, Renormalization and asymptotic expansions, Birkhäuser, Basel, 1991

[13] J.L. Rosner, Ann. Phys. 44 (1967) 11

[14] O.I Zavialov, G.A. Kravtsova and M.A. Malokostov, to be published in Teor. Mat. Fiz. 